La investigación en las facultades de derecho

Research in law universities

\title{
Ernesto Eseverri
}

Catedrático de Derecho Financiero y Tributario

Presidente del Comité no9 Derecho

Y Jurisprudencia de la CNEAI, a diciembre de 2017)

ernesto@ugr.es 


\section{El interés por investigar y la función de la CNEAI}

En líneas generales, la actividad investigadora en la Universidad española se ha duplicado desde el año 2005 con el correspondiente incremento de la producción científica, siendo un exponente de esta afirmación lo que ha sucedido en la Universidad Central o en la Autónoma, ambas de Barcelona, y en la Autónoma de Madrid, si bien, el incremento de productividad en la investigación desde la fecha señalada, ha sido una constante cualquiera que sea la Universidad que se tome en consideración.

También se advierte que el profesorado de nuestras universidades se está viendo sometido a una búsqueda constante de mayor calidad en sus publicaciones, lo que ha provocado un cambio interesante en los hábitos tradicionales de publicación de trabajos científicos que se ha concretado en constantes intentos por publicar en revistas de prestigio y de mayor impacto en el ámbito de la Ciencia Jurídica.

La situación es más plausible, si cabe, teniendo en cuenta que en los últimos tiempos la reducción de recursos a la productividad científica ha sido notable, de modo que si en estos años se ha apreciado un aumento de la producción científica que contrasta con la reducción de recursos económicos para su desarrollo, solo puede ser razonablemente explicado por el interés manifiesto del profesorado universitario por desarrollar una intensa y cuidada actividad investigadora, comportamiento en el que algo han tenido que ver los sistemas de evaluación de la calidad docente e investigadora de dicho profesorado que, al tiempo de incentivar la actividad investigadora del profesorado universitario han supuesto un intento por la búsqueda del "conocimiento válido" o "certificado de calidad" en la investigación, instaurándose en nuestras Universidades una suerte de "cultura de la evaluación"que ha dado nacimiento al concepto del "Estado evaluador" que, en un principio, ha proyectado su actuación sobre el control de los proyectos de investigación otorgados y de los recursos económicos para financiarlos, y más adelante se ha asentado también sobre la evaluación de la actividad investigadora del individuo, naciendo de este modo la Comisión Nacional para la Evaluación de la Actividad Investigadora (CNEAI) junto con las Agencias Evaluadoras Autonómicas, como instrumento que permite acreditar la calidad de la investigación y la experiencia investigadora del evaluado.

\section{2.- Función de la CNEAI}

La función de la CNEAI consiste en llevar a cabo la evaluación de la actividad investigadora de los profesores universitarios, por períodos de seis años, sobre la base de su solicitud voluntaria, con el objetivo, en un principio, de alcanzar un complemento económico de productividad, finalidad que con el discurrir del tiempo se ha visto sobrepasada en cuanto que el reconocimiento positivo de la acción investigadora individual dl profesorado universitario le permite atenuar su carga docente, participar en procesos de selección al acceso de cuerpos docentes universitarios, formar parte de comisiones de tesis doctorales, incluso, de los Comités encargados de la evaluación científica del profesorado de nuestras Universidades. 
Su nacimiento como complemento de productividad, no puede hacernos perder de vista que el reconocimiento positivo de la labor investigadora del profesorado universitario ha de servir, sobre todo, para premiar la calidad de la investigación, aunque tampoco estaría de más reivindicar que no se pierda el incentivo económico, por ejemplo, cuando se alcanza la edad de jubilación.

Entiendo que la función de la CNEAI no debe ser la de sancionar la investigación deficiente, sino estimular el trabajo científico y la calidad de los que se realizan en la Universidad Española. En este sentido, soy del parecer de que una evaluación negativa de los méritos científicos seleccionados por el solicitante es tanto más perjudicial para quien la recibe, no ya por la valoración peyorativa recibida, sino porque no incentiva y desalienta el quehacer cotidiano del investigador. Debemos ser cautos a la hora de emitir esos dictámenes $y$, sin perder el rigor de la exigencia con la que deben revisarse los trabajos que se presentan a evaluación, los encargados de realizarla deben mostrarse comprensivos con ellos procurando el estímulo del investigador, no la crítica despiadada de su trabajo creativo.

Como es conocido en su labor evaluadora la CNEAI se rige por el procedimiento reglado que se recoge en la Orden ministerial de 2 de diciembre de 1994 en la que quedaron fijados una serie de criterios generales para proceder a la evaluación de la actividad investigadora, criterios que pronto se advirtió resultaban de dificultosa aplicación al descender a concretos ámbitos científicos, lo que determinó la formalización de su Anexo por áreas científicas, en lo que hemos convenido en reconocer como requisitos específicos para la evaluación de la producción científica, que anualmente se detallan en sucesivas resoluciones de la CNEAI publicadas en torno al mes de noviembre de cada año.

No obstante, la aplicación de esos criterios -generales y específicos- no tiene un carácter absoluto, pues se modulan atendiendo a las singularidades de cada disciplina, modulación que compete hacer a los correspondientes Comités de expertos que, al finalizar cada uno de los períodos de sexenios solicitados por sus peticionarios, realizan una serie de sugerencias tratando de perfeccionar el sistema de evaluación con la finalidad de hacerlo lo más objetivo posible y eliminando cualquier tipo de discrecionalidad que no venga determinada por la llamada "discrecionalidad técnica" que corresponde a quien valora con criterios científicos ponderables. Naturalmente, las propuestas de los respectivos Comités para que adquieran efectividad, deben ser asumidas en última instancia por el pleno de la CNEAI.

Entre esos criterios específicos que modulan los Comités evaluadores de la actividad científica, se encuentra el de tratar de determinar las condiciones formales que se deben exigir a un medio que difunde los resultados de la investigación para que se pueda esperar un impacto aceptable de esos mismos (revistas científicas especializadas, editoriales, etc.). En este orden de consideraciones, es bueno recordar que el índice de impacto de una aportación científica seleccionada por un profesor universitario para su evaluación, se mide no solo por la calidad de la editorial en que aquella se publica Consejo Asesor, Consejo Editorial, criterios de selección y publicación de los trabajos, etc.- sino además, por el impacto mismo de la aportación publicada, lo que solo es 
mensurable a través de las citas recibidas, dato objetivo que permite ponderar la transcendencia científica de lo publicado a través de quienes se han hecho eco de ella por participar de la misma inquietud intelectual que su autor. (Naturalmente, como todos estamos en el "oficio", también se advierte la posible existencia de "citas interesadas" o realizadas en "deber o atención de reciprocidad", técnica que debiera evitarse en la medida de lo posible).

En cualquiera de los casos, es ineludible señalar que cualquier intento por medir con carácter objetivo la calidad científica de un trabajo está llamado al fracaso, porque su valoración resulta siempre subjetiva, lo que no debe interpretarse como "valoración interesada" en modo alguno, sino como resultado de un análisis individual y particularizado. El interés que mueve a los integrantes de los Comités es solo el deseo de incentivar la actividad investigadora del profesorado universitario y en este sentido con el mismo criterio se ha de medir al novel investigador que se aproxima a la evaluación de su primer sexenio, como al veterano que ya se halla al límite del reconocimiento permitido del incentivo investigador. Al primero, porque quien evalúa está convencido de que el reconocimiento de un primer sexenio ha de servir de estímulo para proseguir tan atractiva y fructífera tarea en prosecución de otros más, y al segundo, porque sirve de estímulo al evaluador que aprecia cómo profesores universitarios con más de treinta y tantos años de investigación continuada a las espaldas, prosiguen en su tarea cotidiana motivados por su trabajo, al margen de cualquier prebenda adjetiva que pueda acompañar a su labor.

Tratando de descender al ámbito propio que nos ocupa, la evaluación de la calidad investigadora en el contexto de la ciencia jurídica presenta una dificultad si se compara con el método de llevarla a cabo en otros ámbitos del saber. Se trata de que en el campo del Derecho no existen índices internacionales que ordenen cuáles son las publicaciones de reconocido prestigio atendiendo al factor impacto (frecuencia con que es citada una revista en un año concreto), índice de inmediatez (rapidez con la que se citan los artículos de un revista), índice $\mathrm{H}$ (que mide la producción científica del investigador y su esfuerzo científico), entre otros más.

Es esta la razón por la que la resolución que establece los criterios específicos para evaluar los trabajos jurídicos, deja abierta a los solicitantes de la evaluación del sexenio la posibilidad de comunicar al Comité las citas de sus trabajos, la relevancia de la investigación seleccionada, y el impacto de las editoriales en que se publica. Criterios que se completan con una serie de requisitos formales adheridos al Anexo I de tales resoluciones, claramente inspirados en los que rigen en aquellas publicaciones que forman parte de los índices internacionales consolidados.

Se advierte entonces, la necesidad de hacer un esfuerzo por establecer esos índices que permitan la evaluación de la actividad investigadora en el ámbito jurídico. Este es el reto que queda por afrontar. 